\title{
Adaptive Crop Monitoring System Based on Wireless Sensor Networks
}

\author{
B. Balaji Bhanu, Mohammed Ali Hussain, Mahmood Ali Mirza
}

\begin{abstract}
Through incorporation of wireless sensor networks (WSNs) into many domains like health care, smart city and various industrial applications, it has become a broad area of research to many experts and scholars worldwide. This paper mainly concentrates on efficient development of agriculture by various modern procedures followed by farmers especially in southern part of India. This includes combination of various climatic parameters like temperature, light, rain and other environmental factors. The existing applications lack location specific data based on their system structure and other key technologies. Therefore there is a need for a systematic architecture based on various locations as environment differs from location to location. This paper presents an adaptive approach for crop management and an easy way to monitor systems which helps the farmer to get a better crop yield. The advantages and challenges of the existing systems are discussed here and various novel and innovative ideas are discussed along with future scope.
\end{abstract}

Keywords: Wireless Sensor Networks (WSN), temperature, light, crop management, environment.

\section{INTRODUCTION}

The Internet of Things (IoT) was first developed by MIT in 1999 with the proposal of RFID Technology. This needs a huge internet-based network which connects various physical and virtual things with different standard and suitable communication protocols. This type of data communication is done by incorporating various wireless sensors and actuators which possess easy exchange of messages or data with each other to facilitate various operations like positioning, tracking identification, monitoring and management [1] with different networks anytime. IoT was applied to many aspects of human life and are connected to various domains related to health care, smart home and so on. It is an ideal option for continuous monitoring and control of different domains especially related to agriculture sector.

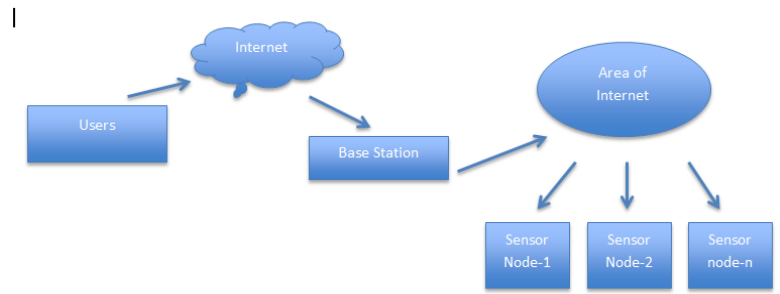

Fig. 1. Wireless Sensor Network (WSNs)

Revised Manuscript Received on December 30, 2019.

* Correspondence Author

B. Balaji Bhanu, Research Scholar Department of CSE, SCSVMV University, Kanchipuram, Tamilnadu, India

Mohammed Ali Hussain, Professor, Department of ECM, KL Deemed to be University, Vaddeswaram, Guntur Dist., AP, India

Mahmood Ali Mirza, Professor, Department of Computer Science Engineering, Krishna University, Machilipatnam, Krishna Dist., AP, India

(c) The Authors. Published by Blue Eyes Intelligence Engineering and Sciences Publication (BEIESP). This is an open access article under the CC BY-NC-ND license (http://creativecommons.org/licenses/by-nc-nd/4.0/)
With the development of WSNs various dependant technologies like cloud computing, machine learning and big data have emerged for facilitating efficient accuracy, handling and retrieval of the sensor data. These technologies play an important role in various aspects of agriculture like helping farmers to monitor soil condition, climate change and plant health. When the climatic parameters change or get disturbed other than the predefined threshold value then the IoT sensors based on wireless network send an alert message to the farmers/administrators based on the server location to remove or change the hidden danger. It can also control various environmental factors like temperature, humidity and carbon dioxide $\left(\mathrm{CO}_{2}\right)$ levels which are necessary for the efficient crop growth in a real time scenario. In addition to the WSNs, cameras are also installed in some places to effectively capture crop other pests related diseases in a real time environment which helps the farmers to easily find the problems and take the necessary preventive action.

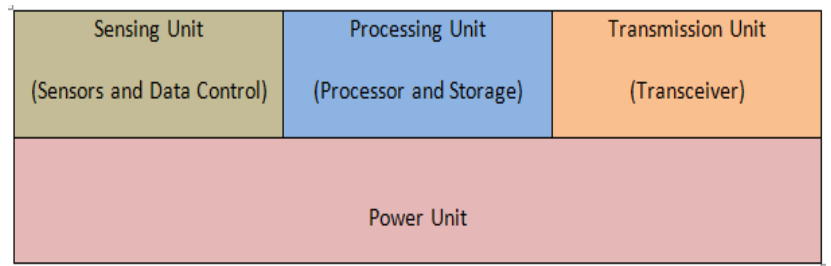

Fig. 2. Sensor Node Architecture

Through various existing technologies such as GPS, RFID and other location based sensors, many goods related to agriculture are being tracked and monitored effectively in a virtual manner during transportation and storage. Farmers use the smart phones or other devices to monitor and predict the status of the crop in a timely manner. This helps to provide various valuable analytics [2] and decisions for various supporting aspects like related to agricultural farms which involves latest technologies like cloud computing, edge computing and many more. The other sections of the paper are discussed followed by the literature survey, proposed methodology and evaluation of the necessary results.

\section{LITERATURE SURVEY}

\section{A. Sensors}

They play an important role in obtaining accurate data from nearby environment to facilitate the development of various IoT based technologies. These sensors include various parameters related to temperature, light intensity, heat, soil, rain, pests and many more.

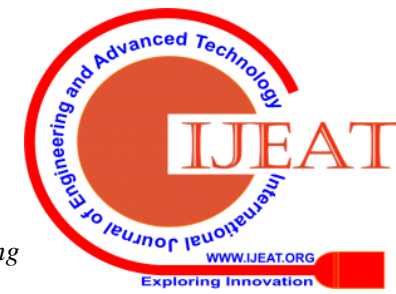




\section{Adaptive Crop Monitoring System Based on Wireless Sensor Networks}

There are many organizations like Honeywell, ENDEVCO and many more which play an important role in deploying and testing these types of sensors at different locations. Generally there are three main types of sensors related to agricultural domain namely physical property type sensor, biosensor and micro electro mechanical system (MEMS) sensors [3]. The physical property type sensor deals with the signal conversion of sensor data based on various parameters like temperature and humidity. The biosensors uses the physical organism as the sensitive component for transmitting information which includes various other sensors like enzyme sensor, microbial and adaptive sensor which are mainly used to detect pesticide quality and quantity on the crop. The mems sensor is an addition to the existing technology where the sensors are in small size, low cost, reliable and which consumes low power. Many researchers have been carried out on using physicochemical properties, spectroscopy, hyper-spectral and nuclear magnetic resonance [4] which facilitates in distributing various physical and chemical properties of soil for efficient crop growth. Many issues related to application of these sensors is still a problem because of lack of various technical standards related to data collection, transmission and cost.

\section{B. Data Transmission}

The real time data is being captured efficiently from different physical sensors and transmitted to various cloud based servers. Due to the technical advancements in the computer networks wireless transmission has been more comfortable to utilize which involves low construction and maintenance, low power consumption and reliability. Many existing research works have been built-up with different WSNs which facilitate environment monitoring for agriculture through remote control process.

\section{Wireless Sensor Networks (WSNs)}

It consists of a lot of sensor nodes that are actually facilitated with efficient sensing, collection and processing of environment data. The data is collected when the sensors are fixed within their range with efficient network coverage. These WSNs are divided into 2 types namely: Terrestrial WSN and Wireless underground sensor networks (WUSN). These sensors are installed in the soil due to low attenuation related to antenna size, cost and energy consumption.

\section{Cloud Computing}

This technology is developed by various combinations of distributed computing, parallel processing and grid computing. It is generally an internet based computing system which provides hardware, platform, software and storage related services to many IoT based applications. It facilitates efficient deployment, allocation and monitoring of various computations which delivers efficient quality of service (QoS). This can provide cheap data storage services to many farmers for various text, image, video and other agricultural information. This facilitates the farmers with various efficient decisions and analytics from the data collected from their agricultural lands. This facilitates with support intelligent computing systems on a large scale which provide a secure platform for various IoT related applications such as crop monitoring.

E. Machine Learning (ML)
It is generally a smart way of computers to simulate various activities for improving performance by acquiring new knowledge. ML has achieved good success which are either theoretical or practical in manner when combined with other agricultural based technologies to maximize crop yield. Some popular algorithms like naive bayes, k-nearest neighbour, support vector machines (SVM), k means, Gaussian mixture models (GMM), deep learning (DL) and so on are generally used for various agricultural applications. ML is applied at different aspects like crop breeding, disease identification and prediction and at various agricultural expert systems for efficient decision making. Many experts came with a method for detecting the maturity of a tomato based on ML which involves 3 steps namely: pixel based segmentation, blob based segmentation and individual fruit detection. The decision trees are generated based on the features such as colour, shape, texture and size are extracted using the first 2 steps. Finally the detected fruit was achieved using $\mathrm{x}$-means clustering method with a recall of 0.80 and precision of 0.88 [5]. Some other researchers proposed a deep learning architecture for the number of fruits based on convolution neural networks. The neural networks are trained using the collected synthetic images to save time. [6].

\section{F. Big Data}

The agricultural sector is applied on various soil databases, environment data and other related data. This technology uses various machine learning, deep neural network algorithms to compute various agricultural models in the form of text, charts, pictures, animations and other multimedia related data. The data computed using various ML algorithms are related with various subdomains like image processing, simulation, statistical analysis and geographical information systems (GIS) [7].

\section{PROPOSED METHODOLOGY}

The existing systems have employed various WSNs for monitoring the soil condition for irrigation which facilitates with analysing the real time data collected from the field. Some of the systems capture the data which controls various sprinkler valves for watering the field. In terms of ML, IoT based on the research towards crop yield [6] and to predict various crop based diseases. The soil condition is computed based on trained data set for automatic irrigation of fields without any human intervention. There is only a M2M system established which interacts between the system for facilitating efficient analysis and predictions.

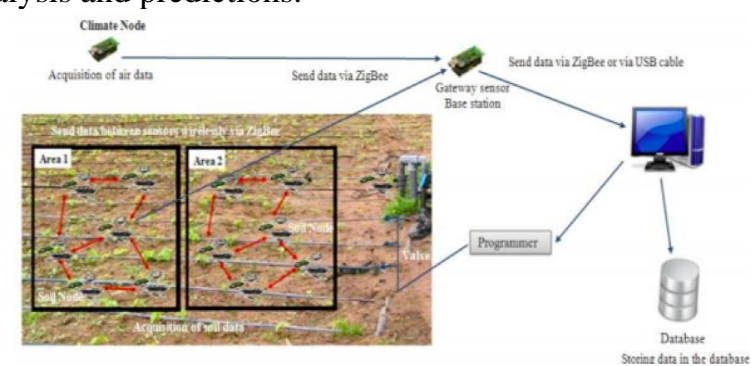

Fig. 3. Proposed Methodology [3] 
Table-I: District-wise productivity in Andhra Pradesh District wise productivity Area

\begin{tabular}{|r|l|r|}
\hline S.No & District & $\begin{array}{l}\text { Highest } \\
\text { productivity (Kg/ } \\
\text { Ha) (approx) }\end{array}$ \\
\hline $\mathbf{1}$ & Ananthapur & 2630 \\
\hline $\mathbf{2}$ & Chittor & 2373 \\
\hline $\mathbf{3}$ & East Godavari & 2978 \\
\hline $\mathbf{4}$ & Guntur & 3239 \\
\hline $\mathbf{5}$ & Kadapa & 2610 \\
\hline $\mathbf{6}$ & Krishna & 3142 \\
\hline $\mathbf{7}$ & Kurnool & 2942 \\
\hline $\mathbf{8}$ & Nellore & 2864 \\
\hline $\mathbf{9}$ & Prakasam & 2985 \\
\hline $\mathbf{1 0}$ & Srikakulam & 1864 \\
\hline $\mathbf{1 1}$ & Visakhapatnam & 1430 \\
\hline $\mathbf{1 2}$ & Vizianagaram & 1957 \\
\hline $\mathbf{1 3}$ & West Godavari & 3322 \\
\hline & & \\
\hline
\end{tabular}

Many problems are considered from the existing systems and are overcome with the development of intelligent IoT based automated irrigation system with various temperature and moisture sensors. This data is transmitted to various edge to edge device connectivity with the help of an IoT board. These boards consider the ML algorithms called KNN which takes the soil moisture and temperature sensors. These algorithms classify the objects based on nearest training samples where the function is employed and deployed locally. The collected data are analysed and updated in the cloud server which lets the farmers know the actual condition of the crop. A graph or any other pictorial representation is obtained as an output by comparing various sensor parameters.

The proposed system architecture comprises of various physical components like Mother Board (Arduino or Raspberry $\mathrm{Pi}$, sensors and many more. These boards are interconnected with various temperatures and soil based sensors which are serially computed and later the algorithms (ML or DL) are applied based on the sensor predicted levels. The obtained output is then predicted for sending the control signal using a serial communication for various sensing process. Below figure shows the complete flow of IoT based automated irrigation system:

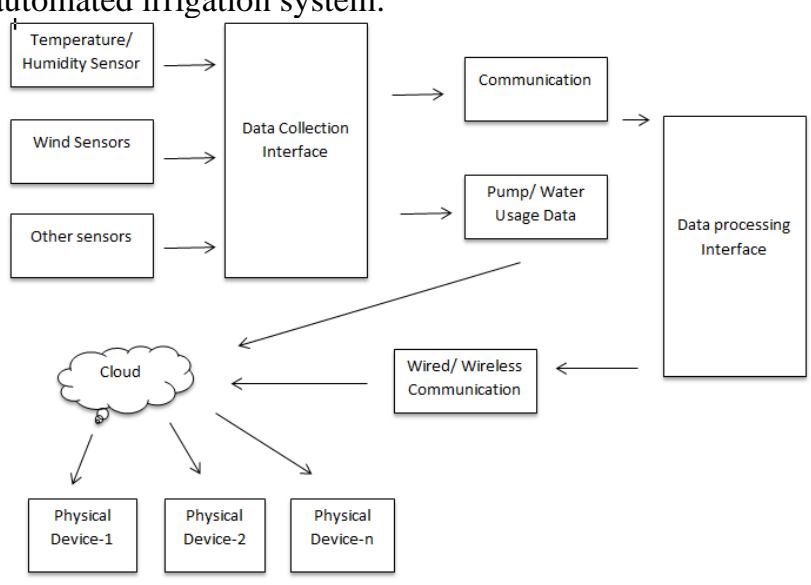

Fig. 4. IoT based Crop Monitoring System

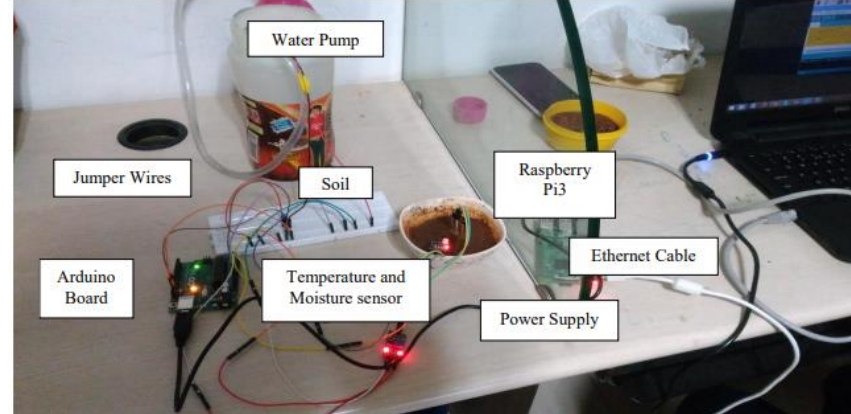

Fig. 5. IoT based irrigation system prototype [5]

Various components used in this system are listed below:

1)Soil Temperature Sensor (STS): This sensor helps in monitoring the temperature of the soil which is available in variety of designs like thermistors and thermocouples. The electric signals transmitted from the sensors to the cloud databases which are later converted to different measurement units like ${ }^{0} \mathrm{C}$ and ${ }^{0} \mathrm{~F}$.

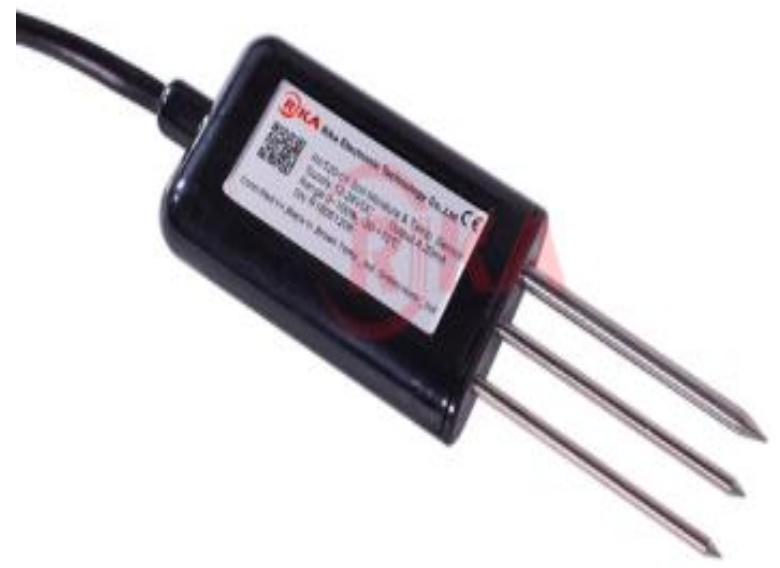

Fig. 6. Soil temperature sensor diagram

2)Soil Moisture Sensor (SMS): The water content in the soil is monitored which required in removing, drying, weighting and evaluating the volumetric water content indirectly by using various soil properties. These are also used to measure water levels which include various tensiometers and gypsum blocks.

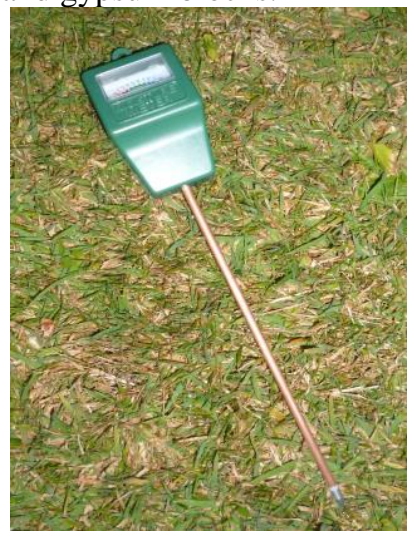

Fig.7. Soil Moisture Sensor

3)Zigbee Module: This technology is based on IEEE standard specification where three zigbee specifications are included namely Xigbee, Zigbee IP and Zigbee RFC.

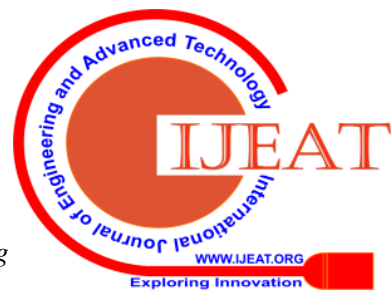




\section{Adaptive Crop Monitoring System Based on Wireless Sensor Networks}

Zigbee optimizes the standard for various networks which best suites for high-level communication protocols which are used to implement at various minor applications like home automation, medical device data collection and low bandwidth devices, which ultimately reduces the power consumption transmission distances. Some other sensors utilized in this research are described below:

Table-II: Different sensors used in this research

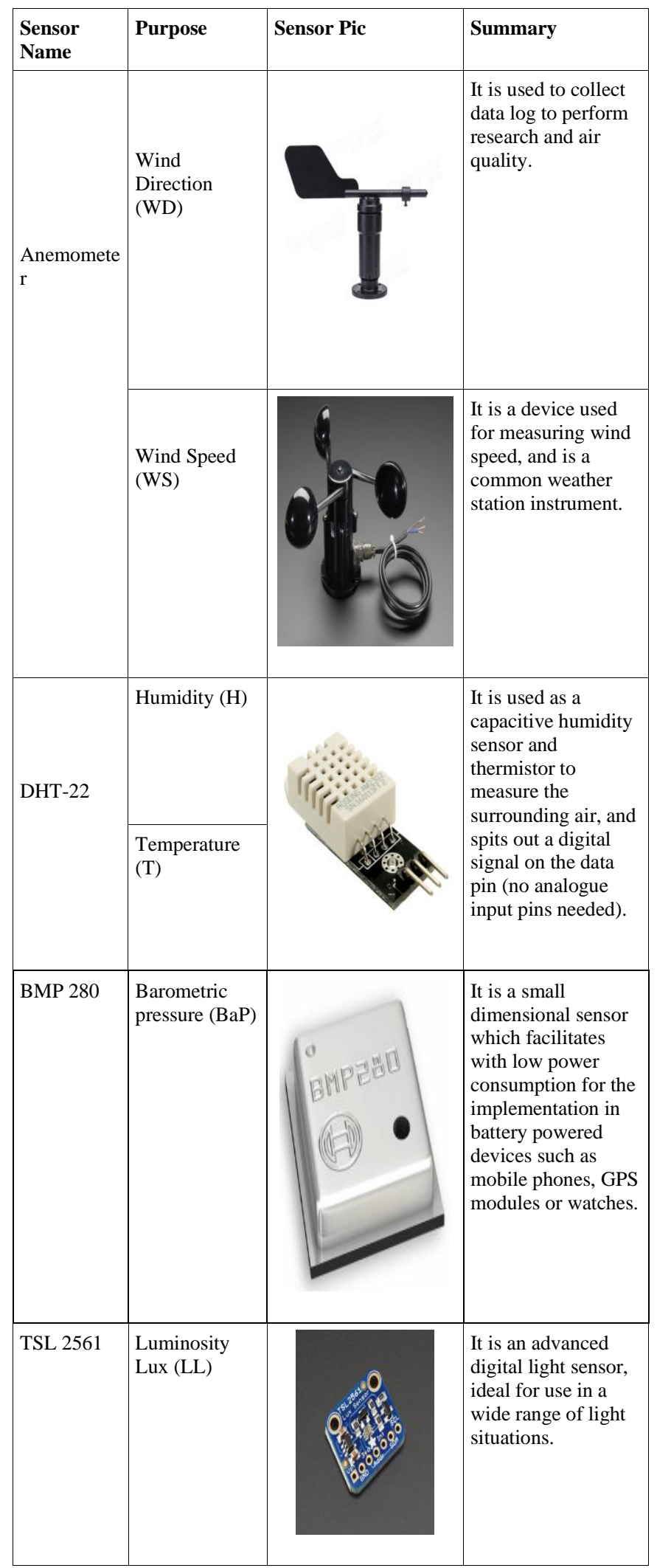

This research work is mainly built on 5 main principles which follows the European MCYFS standards like:

1) An infrastructure to send and retrieve data effectively.

2) Facilitating remote sensing data process.

3) A software monitoring platform for the provided physical infrastructure.

4) Proper monitoring of quantitative and qualitative analysis of crop

5) Proper decision decision-making system for estimating the crop growth and yield.

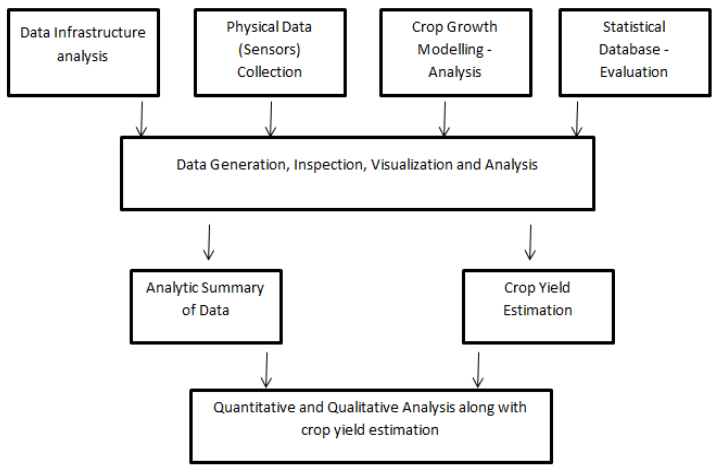

Fig. 8. Overall crop monitoring system structure

\section{EVALUATION}

These crop and farm management techniques are increasingly used by many farm lands across India to achieve sustainable crop production. This process is achieved by implementing a conventional approach based algorithm which results in producing better crop yield which is as follows:

Step:1 - Initialisation of the locations necessary to collect data at crop farms.

Step:2 - Comparing the soil fertility because it depends on several environment factors.

Step:3 - Iterating the soil fertility at various locations in the farm to produce maximum yield.

Step:4 - Identification the data from other sensors

Step:5 - Uploading the data received from the sensors to cloud based servers.

Step:6 - Analysing the data obtained with various parameters which are understandable by the farmers.

Step:7 - Decision making from the analysis done with the previous step and decide the best practise for effective crop yield.

The pseudocode for the above algorithm is as follows: Initialization();

Soil Fertility(Soil_Compare)

For (crop=1; crop<=Max_yield; crop ++ )

\{

Windspeed();

Humidity();

Temperature();

BarometricPressure();

Luminosity();

\}

Output();

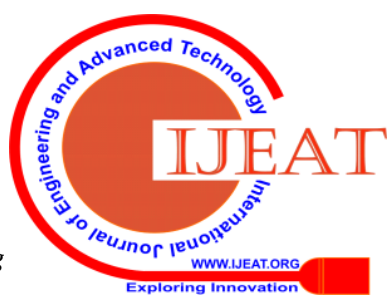


The crop yield is calculated by combining all the sensor parameters which are dependent on individual variables and are responsible for crop yield maximisation aside from the soil fertility factor.

Crop_Yield = f(STS, SMS, PIR, SF, WS, H, T, BaP, LL)

and the regression form of the model is given by

Crop_Yield $=\mathrm{a}_{0}+\mathrm{a}_{1}$ STS $+\mathrm{a}_{2} \mathrm{SMS}+\mathrm{a}_{3} \mathrm{PIR}+\mathrm{a}_{4} \mathrm{SF}+\mathrm{a}_{5} \mathrm{WS}$

$+\mathrm{a}_{6} \mathrm{H}+\mathrm{a}_{7} \mathrm{~T}+\mathrm{a}_{8} \mathrm{BaP}+\mathrm{a}_{9} \mathrm{LL}+\mu----(2)$

where $\mathrm{a}_{1}, \mathrm{a}_{2}, \mathrm{a}_{3}, \mathrm{a}_{4}, \mathrm{a}_{5}, \mathrm{a}_{6}, \mathrm{a}_{7}, \mathrm{a}_{8}$ and $\mathrm{a}_{9}$ are parameters of the model and coefficients of the deployed sensors.

The proposed methodology is tested by using various randomly generated 10 iterative sites where the sensors are deployed at different locations. The maximum yield and maximum fertility are randomly generated in a set $\{100$, simulated and set as follows:

Soil fertility $=100$, Maximum no: of crop yields $=200$, crossover rate $=0.8$, Mutation rate $=0.1$, Penalty factor $\mathrm{M}=$ 0.5

\section{Table III- Tabular summary of farm output}

\begin{tabular}{|r|r|rrr|r|}
\hline & \multicolumn{6}{|c|}{ Summary of Number of Trails } \\
\hline & \multicolumn{5}{|c|}{ Number of Sensor Parameters } \\
\hline Farm Output & 11 & 12 & 9 & 14 & 21 \\
\hline & 1.26 & 1.28 & 1.3 & 1.32 & 1.34 \\
\hline
\end{tabular}

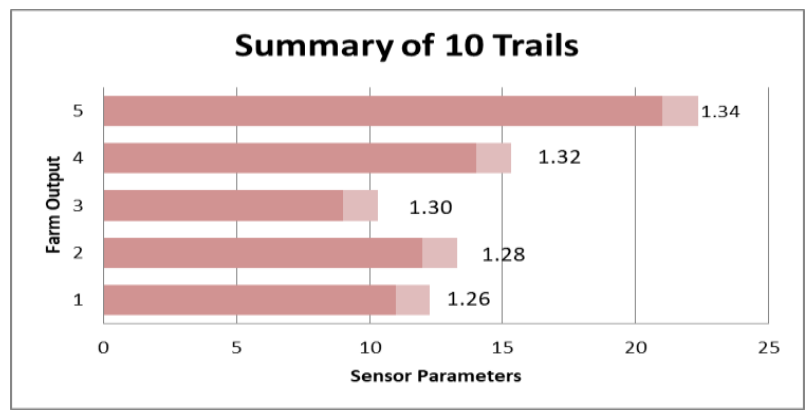

Figure 9. Summary of 10 trails (graph)

The total five experimental work produces 10 different solutions as the crop yield depends on certain amount of constraints like soil fertility, climatic influence which results in various fluctuations in the crop yields obtained by the farm land. The effect of the key parameters (soil fertility and max. number of crop yields) is revealed when these parameters generate either identical or un-identical results.

In the final analysis, the crop yield is dependent on various environmental conditions which affect the interactions between biophysical and various technical components which are unpredictable and random in nature. A series of trails are verified with proposed methodology and was efficient in obtaining the near-optimum solution for this crop management system.

\section{CONCLUSION \& FUTURE WORK}

Although the development of crop monitoring systems utilising various environment friendly sensors many problems exist which are based on various crop and farm conditions. The farmers have to monitor the crop status in a timely manner which produces a better crop yield. Finally $110,120, \ldots .480,490,500)$ and the GA parameters were

through incorporation of various sensors into the crop based management systems there is an increase in the crop yield efficiency.

Many researchers have proposed many crop management systems which are location specific and are adaptable to the climatic conditions at those particular locations. Hence there is a need to work on a generalized crop management system which best suited for any type of farm at any environment condition. Sufficient progress is also required on the development of various quantitative crop monitoring models. As monitoring and assessment of the crop conditions from time to time is estimated which influences crop yield should be effectively implemented which results in producing efficient yield.

\section{REFERENCES}

1. Chief-Electronic Publishing Policy "Increasing crop production sustainably: The perspective of biological processes" (2009) in Food and Agriculture Organization of Europe.

2. Bashir A., Haq S. U., Azhar M., Munir M. A. \& Afzal A. (2012), "Impact of sugarcane Mills Development Activities on Cane Production in Punjab", Pakistan Journal of Agriculture, pp-21-27.

3. Batool S., Habib N., Nazir M., Saddique S., \& Ikram S. (2015), "Trend Analysis of Sugarcane Area and Yield", Technology and Development, pp-46-48.

4. Fernandez A.D.P. \& Nuthall P. L. (2009), "Technical Efficiency in the production of Sugarcane in Central Negros Area, Philippines: An Application of Data Envelopment Analysis", Journal of ISSAAS, pp77-90.

5. Hair J. F., Black W. C., Babin B. J., \& Anderson R. E., (2009), "Multivariate Data Analysis" 7th Edition, Prentice hall.

6. Munir M. A., Hussain M., Imran M. A., Zia S., Anwar H., Ayub M., Rashid M., Jamil I., \& Ghaffar I. (2015), "Analysis of profit Efficiency in Sugarcane Production in District Sargodha, Punjab, Paksitan", International Journal of Eco. Commerce and Management, pp-649-658.

7. Nisha, (2015), “Top 10 Sugarcane Producing Countries", on http://www.perfectinsider.com/top-10-largest-sugarcane-producingcountries/

8. Omotesho O. A.,. Lawal A. M., Olatinwo K. B., Adenuga A.H. \& Bello A. J. (2013), "Technical Efficiency of Sugar Cane Production in Niger State, Nigeria", Journal of Agriculture, Forestry and Social sciences.

9. Roka F. M., Baucum L. E. \& Alvarez J. J. (2009), "Costs and return for sugarcane production on Muck Soils in Southern Florida" University of Florida IFAS Extension, pp-1-14.

10. Aqeel R.,Zafar-Abbasi A., Islam N.,Shaikh Z.A. (2014) "A review of wireless sensors and networks applications in agriculture", Computer Standard Interfaces, pp- 263-270.

11. Ojha T.; Misra S.; Raghuwanshi N.S.,(2015)“Wireless sensor networks for agriculture: The state-of-the-art in practice and future challenges', Computer Electron. Agric., pp-66-84.

12. Jawad H.M.,Nordin R.,Gharghan S.K., Jawad A.M.,Ismail M., (2017), "Energy-efficient wireless sensor networks for precision agriculture: A review", Journal of Sensors, pp-17-81.

13. Talavera J.M., Tobón L.E., Gómez J.A., Culman M.A., Aranda J.M., Parra D.T., Quiroz L.A., Hoyos, A., Garreta L.E. (2017)“Review of IoT applications in agro-industrial and environmental fields", Comput. Electron. Agric. pp-283-297.

14. Tzounis A.,Katsoulas N., Bartzanas T., Kittas C.,(2017) "Internet of things in agriculture, recent advances and future challenges", Biosyst. Eng. pp- 31-48.

15. Ryu M.,Yun J., Miao T., Ahn I.Y., Choi S.C., Kim, (2015) “Design and Implementation of a Connected Farm for Smart Farming System", In Proceedings of the 2015 IEEE SENSORS, Busan, Korea, pp. 1-4.

16. Popović T., Latinović N., Pešić A., Zečević Ž., Krstajić B., Djukanović S. (2017) "Architecting an IoT-enabled platform for precision agriculture and ecological monitoring: A case study", Comput. Electron. Agric. pp- 255-265.

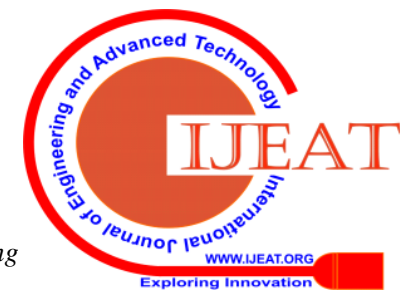


17. Playán E., Salvador R. Bonet L., Camacho E., Intrigliolo D.S., Moreno M.A., Rodríguez-Díaz J.A., Tarjuelo J.M., Madurga C.; Zazo T., (2018) "Assessing telemetry and remote control systems for water users associations in Spain”, Agric. Water Manag. pp- 89-98.

\section{AUTHORS PROFILE}

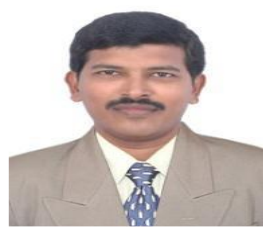

B. Balaji Bhanu, received his M.Sc. Electronics, MCA, M.Tech. in CSE, M.Phil. in Electronic instrumentation. He is currently a Ph.D. candidate at SCSVMV University \& presently working as a research scholar. He is having a teaching experience of many years in teaching for graduates and post graduates. He published number of papers at national and international conferences along with a peer reviewed journals. His main research interests include Wireless Sensor Networks and IoT.

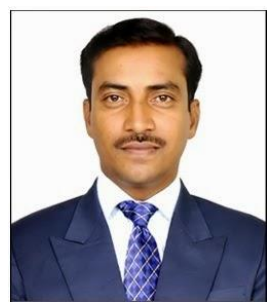

Dr. Mohammed Ali Hussain, working as Professor in Department of Electronics and Computer Engineering, KL Deemed to be University, Guntur Dist., Andhra Pradesh, India. He received 13 National Awards and 2 International Awards for his research contributions in various International Journals (Scopus \& SCI). He is Editorial Board Member \& Reviewer of various International Journals. He has published 7 patents to his credit and produced $10 \mathrm{PhD}$ 's under his supervision. His area of Interest includes Wireless Networks, Mobile Ad hoc Networks and Web Security. He is a member of various professional bodies FISEEE, ASDF, UACEE, IACSIT and IAENG.

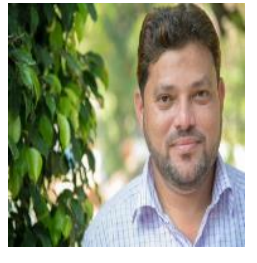

Dr. Mahmood Ali Mirza ,working as Professor in Department of Computer Science, Krishna University, Krishna Dist., Andhra Pradesh, India. He published large number of papers in Scopus \& SCI Journals. He is Active reviewer of IEEE, Springer, and Elsevier Journals. His Area of Interest includes Data Mining, Machine Learning and Networks. 